\title{
Rupture Cascades in a Discrete Element Model of a Porous Sedimentary Rock
}

\author{
Ferenc Kun, ${ }^{1}$ Imre Varga, ${ }^{1}$ Sabine Lennartz-Sassinek, ${ }^{2,3}$ and Ian G. Main ${ }^{2}$ \\ ${ }^{1}$ Department of Theoretical Physics, University of Debrecen, P.O. Box 5, H-4010 Debrecen, Hungary \\ ${ }^{2}$ School of Geosciences, University of Edinburgh, EH9 3JW Edinburgh, United Kingdom \\ ${ }^{3}$ Institute for Geophysics and Meteorology, University of Cologne, Cologne, Germany \\ (Received 3 May 2013; revised manuscript received 21 August 2013; published 14 February 2014)
}

\begin{abstract}
We investigate the scaling properties of the sources of crackling noise in a fully dynamic numerical model of sedimentary rocks subject to uniaxial compression. The model is initiated by filling a cylindrical container with randomly sized spherical particles that are then connected by breakable beams. Loading at a constant strain rate the cohesive elements fail, and the resulting stress transfer produces sudden bursts of correlated failures, directly analogous to the sources of acoustic emissions in real experiments. The source size, energy, and duration can all be quantified for an individual event, and the population can be analyzed for its scaling properties, including the distribution of waiting times between consecutive events. Despite the nonstationary loading, the results are all characterized by power-law distributions over a broad range of scales in agreement with experiments. As failure is approached, temporal correlation of events emerges accompanied by spatial clustering.
\end{abstract}

Understanding the processes that lead to catastrophic failure of porous granular media is an important problem in a wide variety of applications, notably in Earth science and engineering [1-8]. Such failure is often preceded by detectable changes in mechanical properties (stress and strain) and in geophysical signals (elastic wave velocity, electrical conductivity, and acoustic emissions) measured remotely at the sample boundary [9]. In particular, acoustic emissions result from sources of internal damage due to sudden local dislocations in the form of tensile or shear microcracks whose origin time, location, orientation, duration, and magnitude can all be inferred from the radiated wave train [10]. Typically, only a very small proportion of the microcracks revealed by destructive thin sectioning after the test result in detectable acoustic emissions [11]. As a consequence, experimental data provide only limited insight into the complexity of the microscopic processes at work prior to failure, notably the probability distributions of the relevant parameters, their scaling properties, and their population dynamics.

Theoretical approaches to the dynamics and statistics of rupture cascades have typically been based on stochastic fracture models comprising lattices of springs [12], beams $[13,14]$, fuses $[15,16]$, or fibers [17-19]. However, such lattice models involve a strong simplification of the material microstructure and the inhomogeneous stress field. For example, macroscopic laws of damage for cohesive elements are often implemented at the mesoscopic scale on a regular two-dimensional grid, avoiding the truly threedimensional microstructure of real porous media, and often using power-law rheology as an input. Here, we adopt a discrete element modeling (DEM) approach that relaxes all of these restrictions and allows a realistic investigation of the emergent properties of the dynamics, including the temporal and spatial statistics of the resulting crackling noise. Starting from the level of single particles of porous granular media, rupture cascades and scaling laws both emerge spontaneously in the competition between realistic structural disorder and the interactions and correlations that arise from external dynamic loading and internal stress redistribution. Our approach quantitatively reproduces the observed scaling laws of crackling noise remarkably well without tuning $[9,10,20]$, including those of parameters such as burst energy and duration not available to lattice-based models.

In the model, cylindrical samples are constructed by sedimenting spherical particles in a container. Figure 1(a) illustrates that particles fall one by one on the top of the growing particle layer and dissipate their kinetic energy by colliding with other particles and also with the container wall. The radius of particles $R$ was sampled from a lognormal distribution $\left.p(R) \sim \exp \left[-(\ln R-\ln \bar{R})^{2}\right) /\left(2 \sigma_{R}^{2}\right)\right]$, as shown in Fig. 1(b), which describes the statistics of large particle sizes for various types of Earth materials (see e.g., the particle size distribution prior to faulting in Fig. 7 of Ref. [20]). In order to avoid numerical problems of too wide size distributions, we set the range $R_{\max } / R_{\min }=20$ fixed and choose $\bar{R}=5 R_{\min }$ to have the maximum of $p(R)$ nearly in the middle of the $\left[\log R_{\min }, \log R_{\max }\right]$ interval. The diameter $D_{0}$ and height $H_{0}$ of the cylinder were set to $D_{0}=$ $438.57 R_{\min }$ and $H_{0}=1008.71 R_{\min }$, which yields an aspect ratio $H_{0} / D_{0} \approx 2.3$ as in the experiments of Ref. [20]. With this geometrical setup, the number of particles $N$ of the samples fluctuates in a narrow interval around $N=20000$ with a total porosity $\Phi \approx 56 \%$. Particles lying on the sample surface typically have only a few contacts $n_{c}=1,2,3$ to other ones, while bulk particles are characterized by higher 

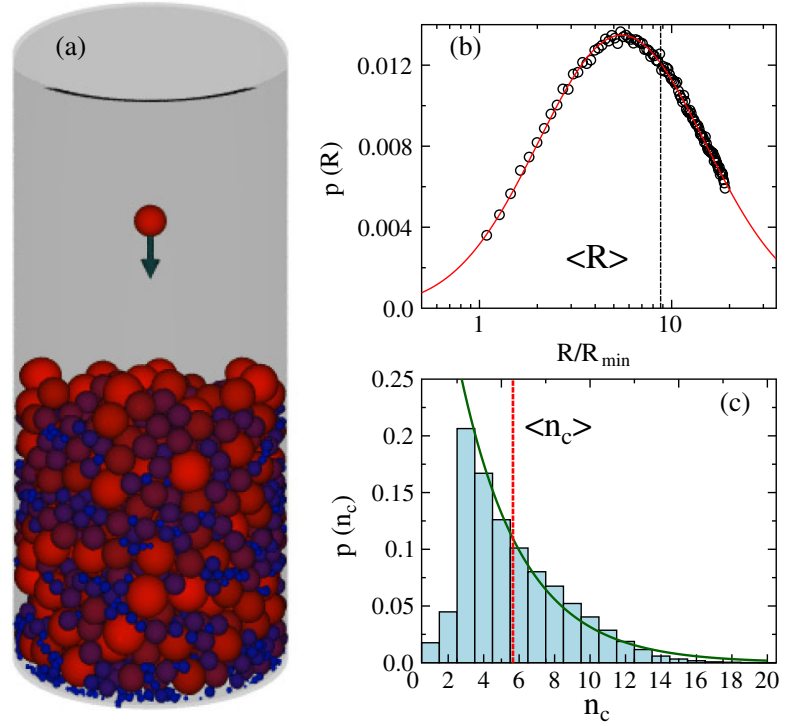

FIG. 1 (color online). (a) Preparation of the sample by sedimenting spherical particles with randomly distributed radius $R$. The color code corresponds to the radius of the particles: the smallest particles are dark blue whereas the biggest ones have light red color. (b) Comparison of the radius distribution $p(R)$ obtained numerically (symbols) to the desired log-normal functional form (continuous line). (c) Histogram $p\left(n_{c}\right)$ of the number of contacts $n_{c}$ in a sample of $N=20000$ particles. Exponential function (green line) was fitted for $n_{c}>3$.

contact numbers. The probability distribution (PDF) $p\left(n_{c}\right)$ of the number of contacts $n_{c}$ proved to be an exponential for $n_{c}>3$, as illustrated in Fig. 1(c). Both the exponential form of $p\left(n_{c}\right)$ and the value of the average number of contacts $\left\langle n_{c}>\approx 5.8\right.$ of our packing are in a reasonable agreement with measurements on porous sandstones [20].

To form a particulate solid in a DEM framework $[13,14,21,22]$, cohesive interaction is provided by beams that connect the particles along the edges of a Delaunay tetrahedrization performed with the initial position of particles. Conceptually, the beam represents the effect of cementation and induration between particles. Beams can suffer elongation, compression, shear, and torsion representing the forces and torques that emerge between interacting particles $[13,14,22]$. The time evolution of the system is followed by molecular dynamics simulations solving the equation of motion of the particles. Beams break when over stressed, according to $[13,14]$

$$
\left(\frac{\varepsilon_{i j}}{\varepsilon_{\mathrm{th}}}\right)^{2}+\frac{\max \left(\left|\Theta_{i}\right|,\left|\Theta_{j}\right|\right)}{\Theta_{\mathrm{th}}}>1,
$$

where $\varepsilon_{i j}$ denotes the axial strain, while $\Theta_{i}$ and $\Theta_{j}$ are the generalized bending angles of the two ends of the beam connecting particles $i$ and $j$. The first and second terms of Eq. (1) represent the contributions of stretching and bending, respectively, where bending mainly arises due to the shear of the particle contacts $[13,14,23]$. In the model, there is only structural disorder present; i.e., the breaking thresholds are set to constant values $\varepsilon_{\text {th }}=0.003$ and $\Theta_{\text {th }}=2^{\circ}$ for all the beams. Those particles that are not connected by beams (e.g., along cracks) interact via Hertz contacts [22].

To simulate uniaxial compression of sedimentary rocks in a strain controlled way, two particle layers on the top and bottom of the cylindrical sample were clamped such that the bottom layer was fixed while the one on the top was moving downward at a constant speed (see the inset of Fig. 2 for illustration). The strain rate $\dot{\varepsilon}$ of loading was set as $\dot{\varepsilon} \Delta t=1.8 \times 10^{-7}$, where $\Delta t$ is the time step used to integrate the equation of motion. The constitutive curve $\sigma(\varepsilon)$ of the system is presented in Fig. 2 where the simulation was stopped when the axial stress $\sigma$ dropped to zero. The system has a highly brittle response: for small deformations linearly elastic behavior is obtained; stronger nonlinearity of $\sigma(\varepsilon)$ is only observed in the vicinity of the maximum $\sigma_{c}$. Macroscopic failure is indicated by a sudden drop of the stress beyond the strain at peak stress $\varepsilon_{c}$.

In the simulations, the breaking criterion of Eq. (1) is evaluated in each iteration step of the equation of motion such that those beams that fulfill the condition are removed and their breaking time $t_{i}^{b}$ is recorded. During the loading process, first the weakest beams break randomly all over the sample due to the quenched disorder starting at $\varepsilon \approx$ 0.0015 in Fig. 2, i.e., relatively early in the loading history. Each breaking event is followed by the redistribution of stress that may induce additional breakings and in turn can even trigger an entire avalanche of beam breakings. If two consecutive beam breakings at times $t_{i}^{b}$ and $t_{i+1}^{b}$ occur within the correlation time $t_{c}$, i.e., $\left|t_{i+1}^{b}-t_{i}^{b}\right|<t_{c}$, they are considered to belong to the same burst. The value of $t_{c}$ was set to $t_{c}=25 \Delta t$, which is approximately the time needed for the elastic waves to pass the radius of the sample $D_{0} / 2$.

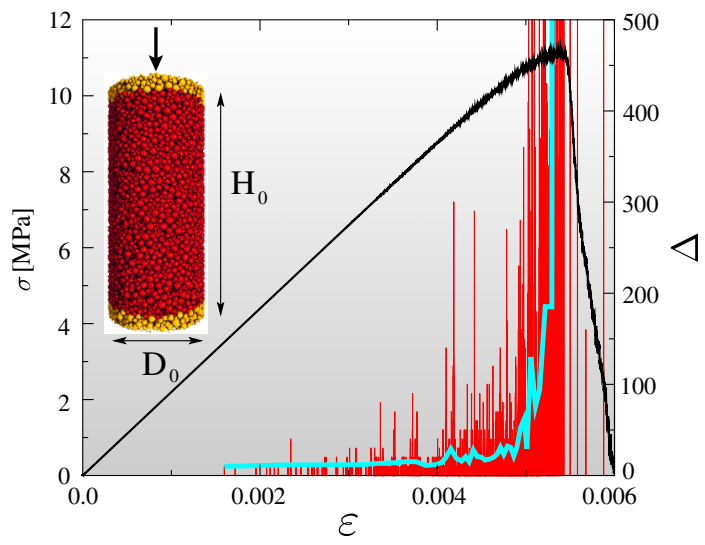

FIG. 2 (color online). Constitutive behavior $\sigma(\varepsilon)$ of a single sample together with the series of burst size $\Delta$ (red bars) as a function of the strain of their appearance. The moving average of $\Delta$ (blue line) was calculated over 50 consecutive events. The inset illustrates the loading condition. 
Similar criteria are also necessary to define real acoustic emission events in laboratory experiments $[3,5,11]$. The breaking bursts of our DEM are analogous to the acoustic emissions generated by the nucleation and propagation of cracks in laboratory experiments on geomaterials and in field observations on geological faults [3,5,11]. We define the burst size $\Delta$ as the number of beams breaking in the correlated sequence, which is related to the rupture area created by the burst. Figure 2 shows that despite the smooth macroscopic response $\sigma(\varepsilon)$ of the system the size of bursts $\Delta$ exhibits strong fluctuations while its average increases as the maximum of $\sigma(\varepsilon)$ is approached. At the beginning of the breaking process, only small bursts of a few breaking beams appear; however, as loading proceeds the triggering of longer avalanches becomes more probable. Strong bursting activity with complex structure of the event series emerges after $\sigma$ exceeds approximately the two-third level of the peak stress $\sigma_{c}$ in agreement with experiments [20]. The total number of bursts we identify during the fracture of a single sample is about 2000-2200.

Since the dynamics of the breaking process changes at the peak load, we analyzed the statistical features of the time series of bursts separately for events occurring before $\varepsilon<\varepsilon_{c}$ and after $\varepsilon>\varepsilon_{c}$ the peak of $\sigma(\varepsilon)$. Figure 3(a) shows that the PDF of burst sizes $p(\Delta)$ of prepeak events has a power-law functional form followed by a cutoff with stretched exponential shape

$$
p(\Delta) \sim \Delta^{-\tau} \exp \left[-\left(\Delta / \Delta^{*}\right)^{c}\right] .
$$

A high-quality fit was obtained with a rupture size exponent $\tau=2.22$, whereas the cutoff parameters are $c=$ 1.5 and $\Delta^{*}=1200$. At the peak of the constitutive curve
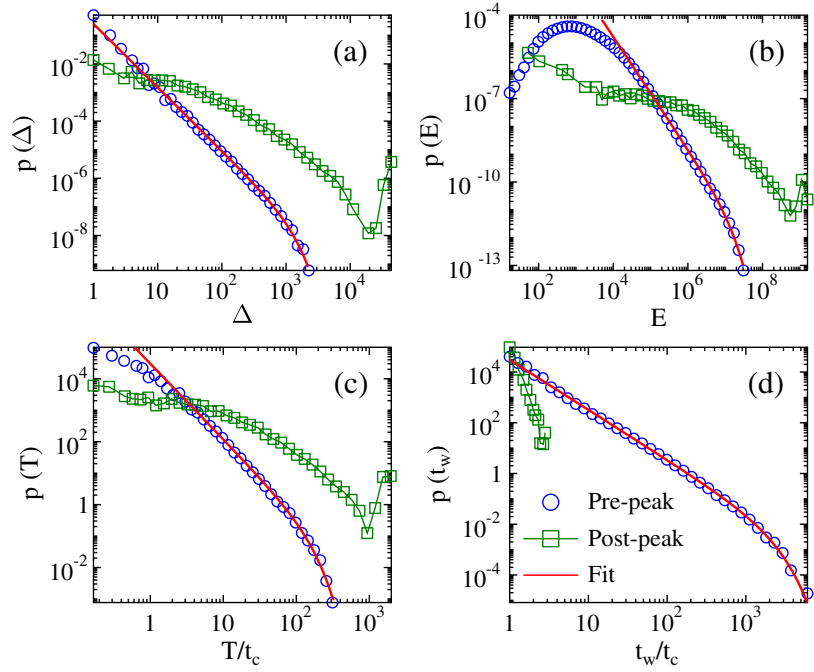

FIG. 3 (color online). Probability distributions of the characteristic quantities of bursts occurring before and after the peak of $\sigma(\varepsilon)$ : distribution of burst (a) size $p(\Delta)$, (b) energy $p(E)$, (c) duration $p(T)$, and (d) waiting time $p\left(t_{w}\right)$. The red lines represent fits with the functional form of Eq. (2). $\sigma(\varepsilon)$, the dynamics of the rupture process undergoes bifurcation, indicated by the different statistics of postpeak events in Fig. 3(a). Although only a small fraction 3\% of the bursts (about $12 \%$ of broken beams) occurs along the softening branch of $\sigma(\varepsilon)$, large avalanches are more frequent in this regime. The small hump of the largest events corresponds to the final multifragmentation of the sample. As the burst is formed, the elastic energy $E_{j}^{b}$ stored in beams is released, which can be directly compared to the energy of acoustic signals in experiments. The overall duration $T$ of a burst is the difference of the time of the first and last beam breaking in the correlated sequence $T=t_{\Delta}^{b}-t_{1}^{b}$. Figures 3(b) and 3(c) show that in the prepeak regime the PDF of burst energy $p(E)$ and duration $p(T)$ both have a power-law decay with a stretched exponential cutoff similar to the behavior of the burst size [Eq. ((2)]. Best fits were obtained with the power-law exponents $\alpha=$ 2.02 and $\beta=2.4$ for the burst energy and duration, respectively, while the cutoff parameters are $c_{E}=1.0$, $E^{*}=1.1 \times 10^{7}$ and $c_{T}=1.5$, and $T^{*} / t_{c}=170$. The corresponding distributions of postpeak event source parameters (size, energy, and duration) share a similar qualitative shape, with a break of slope at low magnitude and a bump at high values, the latter likely associated with the finite sample size.

Bursts are separated by silent periods where no beam breaking occurs. The duration $t_{w}$ of these interevent periods encodes interesting information about the temporal dynamics of fracture. The minimum value of $t_{w}$ is determined by the correlation time $\min \left(t_{w}\right) \approx t_{c}$; hence, in Fig. 3(d) the PDF of waiting times $p\left(t_{w}\right)$ is presented as a function of the dimensionless ratio $t_{w} / t_{c}$ [the same is applied for $T$ in Fig. 3(c)] Again, the same functional form of Eq. (2) of the distribution is evidenced where the best fit was obtained with the power-law exponent $z=2.0$, whereas the cutoff parameters are obtained as $c_{w}=1.2$ and $t_{w}^{*} / t_{c}=1600$. Note that the separating postpeak events have only a minor effect on $p\left(t_{w}\right)$ since bursts in the postpeak regime occur rapidly with very short waiting times. We emphasize that the exponents $\tau, \alpha, \beta$, and $z$ of the distributions are robust with respect to the correlation time $t_{c}$ in the range $20 \Delta t<t_{c}<35 \Delta t$, i.e., until $t_{c}$ falls close to the time the elastic wave takes to cross the sample. Only the cutoffs of the distributions change.

Characteristic quantities of single bursts $\Delta, E$, and $T$ are not independent of each other: large bursts typically release a higher amount of energy and have a longer duration. In order to quantify these correlations, we determined the average duration $\langle T\rangle$ and energy $\langle E\rangle$ of bursts as a function of their size $\Delta$ separately for pre- and postpeak events. In Figure 4(a) a strong correlation is observed with power-law functional forms

$$
\langle E\rangle \sim \Delta^{\nu_{E}} \quad \text { and } \quad\langle T\rangle \sim \Delta^{\nu_{T}} .
$$



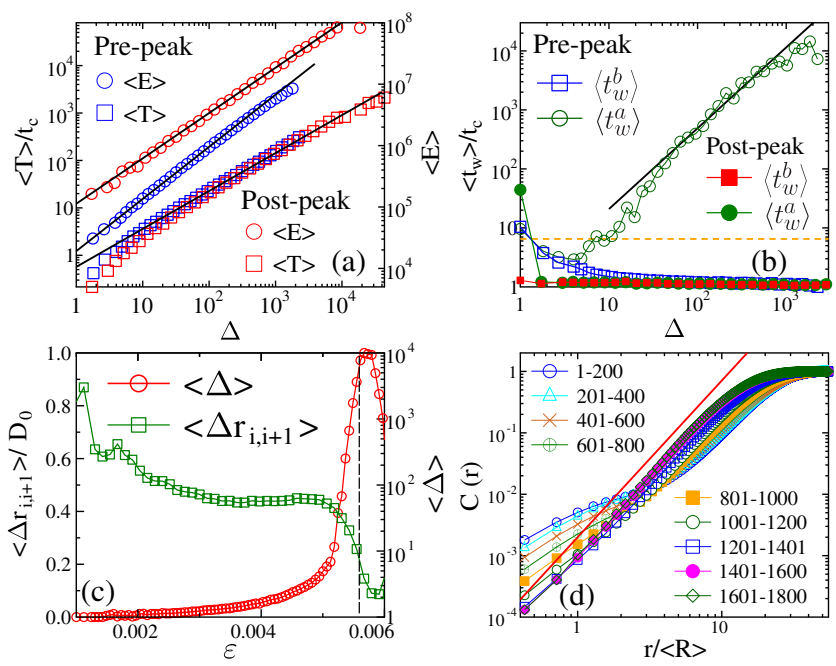

FIG. 4 (color online). (a) The average energy and duration of bursts as function of their size. (b) Average waiting times separately calculated before $\left\langle t_{w}^{b}\right\rangle$ and after $\left\langle t_{w}^{a}\right\rangle$ events as a function of $\Delta$. The straight lines in (a) and (b) are fits with Eqs. (3) and (4). (c) Average distance between consecutive events and the average size of bursts as function of strain. The vertical line indicates the peak position of $\sigma(\varepsilon)$. (d) The correlation integral $C(r)$ for windows of 200 consecutive events.

The duration of bursts has the same behavior in both the pre- and postpeak regimes with a unique exponent $\nu_{T}=0.8$. However, the energy of bursts of the same size proved to be higher for post- than for prepeak events, since they are formed by the breaking of stronger beams. The released energy is nearly proportional to the burst size with exponents $\nu_{E}=1.15$ and $\nu_{E}=1.0$ for $\varepsilon<\varepsilon_{c}$ and $\varepsilon>\varepsilon_{c}$, respectively. These are lower than the scaling exponent of 1.5 commonly inferred from a simple dislocation theory for the seismic source in interpreting laboratory acoustic emission data [24]. Equations (3) yield relations between the prepeak exponents $\alpha=\left(\tau+\nu_{E}-\right.$ $1) / \nu_{E}$ and $\beta=\left(\tau+\nu_{T}-1\right) / \nu_{T}$, in good agreement with our numerically determined exponents.

The stress redistribution around cracks gives rise to correlations between bursts that become more and more relevant as the system approaches failure. To obtain information about how events affect the appearance of later bursts, we determined the average value of waiting times as a function of the burst size $\Delta$ separately averaging $t_{w}$ that elapsed before $t_{w}^{b}$ and after $t_{w}^{a}$ the events. Since along the softening branch of $\sigma(\varepsilon)$ the specimen is collapsing with large bursts, in the postpeak regime both $\left\langle t_{w}^{a}\right\rangle$ and $\left\langle t_{w}^{b}\right\rangle$ rapidly converge to the vicinity of the most probable waiting time $t_{c}$ [see also Fig. 3(d)] indicating the absence of correlations. In the prepeak regime $\left\langle t_{w}^{b}\right\rangle$ has the same behavior though the convergence is slower. The most remarkable result is that $\left\langle t_{w}^{a}\right\rangle$ increases for large event sizes according to

$$
\left\langle t_{w}^{a}\right\rangle \sim \Delta^{\nu_{w}}
$$

with the exponent $\nu_{w}=1.37$. This correlation arises because a larger burst releases stress in a larger volume of the specimen so that it requires a longer time to build up the stress again and to trigger the next burst. Our calculations revealed that the emergence of temporal correlations is also accompanied by spatial clustering of events. Figure 4(c) presents the average distance of consecutive bursts $\left\langle\Delta r_{i, i+1}\right\rangle=\sqrt{\left\langle\left(\vec{r}_{i+1}-\vec{r}_{i}\right)^{2}\right\rangle}$ as a function of strain $\varepsilon$, where the position $\vec{r}_{i}$ of a single burst is identified by the center of mass of its broken beams. For a broad range of $\varepsilon$, the ratio $\left\langle\Delta r_{i, i+1}\right\rangle / D_{0}$ falls close to 0.5 , which implies that events randomly scatter all over the sample. However, approaching the peak load $\sigma_{c}$ the distance rapidly decreases, which clearly marks spatial clustering of events. In Fig. 4(c) the average size of bursts $\langle\Delta\rangle$ increases with $\varepsilon$ and reaches a maximum slightly beyond the peak of the consecutive curve. At the strain where spatial correlation sets on, $\langle\Delta\rangle$ switches to a faster growth. A more detailed measure of spatial correlation is provided by the correlation integral $C(r)$ defined as $C(r)=N(<r) / N_{p}$, where $N(<r)$ denotes the number of pair of events with a distance smaller than $r$ and $N_{p}$ is the total number of pairs. To quantify how correlations evolve, we evaluated $C(r)$ for windows of 200 consecutive events. Figure 4(d) shows that approaching failure the correlation integral saturates earlier and for the last four windows it becomes a power law $C(r) \sim r^{D_{2}}$ with the exponent $D_{2}=2.55$, which indicates strong spatial clustering of bursts.

In conclusion, we have successfully reconstructed a synthetic model of the compressive failure of sedimentary rocks with realistic microstructure, breaking dynamics, and loading conditions relevant for catastrophic failure in porous granular media. The statistical properties of the local microcrack events show qualitative agreement with those inferred from acoustic emissions generated under compression in laboratory tests, notably the power-law scaling of the PDFs of rupture area, duration, energy, and waiting time and powerlaw scaling between rupture energy and duration with respect to source size [6-8,24,25]. In recent laboratory experiments on porous rocks and on synthetic samples with well controlled porosity, $\Phi$ power-law distribution of the energy of acoustic events was found with an exponent that increases with $\Phi$ from 1.6 to $2.0[7,8]$. Our simulations have good qualitative agreement with the time evolution of rupture $[7,8]$ and quantitative agreement with the energy exponent [8]. Our simulations also revealed microscopic details of the rupture process, including the temporal evolution of spatial correlations in rupture location that controls the emergence of localized damage at a resolution not readily accessible by experimental means, with potential implications for developing predictive models for catastrophic failure in porous granular media.

We thank the projects TAMOP-4.2.2.A-11/1/KONV2012-0036, TAMOP-4.2.2/B-10/1-2010-0024, OTKA 
K84157, and ERANET_HU_09-1-2011-0002. This work was supported by the European Commissions by the Complexity-NET pilot project LOCAT.

[1] C. Sammis, G. King, and R. Biegel, Pure Appl. Geophys. 125, 777 (1987).

[2] R. L. Biegel, C. G. Sammis, and J. H. Dieterich, J. Struct. Geol. 11, 827 (1989).

[3] P. R. Sammonds, P. G. Meredith, and I. G. Main, Nature (London) 359, 228 (1992).

[4] S. Steacy and C. Sammis, Nature (London) 353, 250 (1991).

[5] M. Heap, P. Baud, P. Meredith, S. Vinciguerra, A. Bell, and I. Main, Earth Planet. Sci. Lett. 307, 71 (2011).

[6] J. Baró, A. Corral, X. Illa, A. Planes, E. K. H. Salje, W. Schranz, D. E. Soto-Parra, and E. Vives, Phys. Rev. Lett. 110, 088702 (2013).

[7] E. Salje, G. Lampronti, D. Soto-Parra, J. Baró, A. Planes, and E. Vives, Am. Mineral. 98, 609 (2013).

[8] P. Castillo-Villa, J. Baró, A. Planes, E. Salje, P. Sellappan, W. Kriven, and E. Vives, J. Phys. Condens. Matter 25, 292202 (2013).

[9] C. H. Scholz, J. Geophys. Res. 73, 1417 (1968).

[10] C. C. Graham, S. Stanchits, I. G. Main, and G. Dresen, Int. J. Rock Mech. Min. Sci. Geomech. Abstr. 47, 161 (2010).

[11] D. Lockner, Int. J. Rock Mech. Min. Sci. Geomech. Abstr. 30, 883 (1993).
[12] L. Girard, J. Weiss, and D. Amitrano, Phys. Rev. Lett. 108, 225502 (2012).

[13] H. A. Carmona, F. K. Wittel, F. Kun, and H. J. Herrmann, Phys. Rev. E 77, 051302 (2008).

[14] G. Timár, J. Blömer, F. Kun, and H. J. Herrmann, Phys. Rev. Lett. 104, 095502 (2010).

[15] M. J. Alava, P. K. V. V. Nukala, and S. Zapperi, Phys. Rev. Lett. 100, 055502 (2008).

[16] M. Alava, P. K. Nukala, and S. Zapperi, Adv. Phys. 55, 349 (2006).

[17] K. Z. Nanjo and D. L. Turcotte, Geophys. J. Int. 162, 859 (2005).

[18] F. Kun, H. A. Carmona, J. S. Andrade, and H. J. Herrmann, Phys. Rev. Lett. 100, 094301 (2008).

[19] S. Pradhan, A. Hansen, and B. K. Chakrabarti, Rev. Mod. Phys. 82, 499 (2010).

[20] K. Mair, I. Main, and S. Elphick, J. Struct. Geol. 22, 25 (2000).

[21] D. Potyondy and P. Cundall, Int. J. Rock Mech. Min. Sci. 41, 1329 (2004).

[22] T. Pöschel and T. Schwager, Computational Granular Dynamics (Springer, Berlin, 2005).

[23] G. A. D’Addetta, F. Kun, and E. Ramm, Granular Matter 4, 77 (2002).

[24] C. G. Hatton, I. G. Main, and P. G. Meredith, J. Struct. Geol. 15, 1485 (1993).

[25] I. Ojala, B. T. Ngwenya, I. G. Main, and S. C. Elphick, J. Geophys. Res. 108, 2268 (2003). 